\title{
FY-93 Noncontacting Acoustic Ultrasonic Signature Analysis Development
}

\author{
D. M. Tow \\ J. G. Rodriguez \\ R. L. Williamson \\ L. G. Blackwood
}

Published April 1994

Idaho National Engineering Laboratory .

EG\&G Idaho, Inc.

Idaho Falls, Idaho 83415

Prepared for the

U.S. Department of Energy

Assistant Secretary for

Office of Nonproliferation and National Security

Under DOE Idaho Operations Office

Contract DE-AC07-76ID01570 


\section{DISCLAIMER}

This report was prepared as an account of work sponsored by an agency of the United States Government. Neither the United States Government nor any agency thereof, nor any of their employees, make any warranty, express or implied, or assumes any legal liability or responsibility for the accuracy, completeness, or usefulness of any information, apparatus, product, or process disclosed, or represents that its use would not infringe privately owned rights. Reference herein to any specific commercial product, process, or service by trade name, trademark, manufacturer, or otherwise does not necessarily constitute or imply its endorsement, recommendation, or favoring by the United States Government or any agency thereof. The views and opinions of authors expressed herein do not necessarily state or reflect those of the United States Government or any agency thereof. 


\section{DISCLAIMER}

Portions of this document may be illegible in electronic image products. Images are produced from the best available original document. 


\section{ABSTRACT}

A noncontacting, long-standoff inspection system with proven capabilities in container fill identification has been under development at the Idaho National Engineering Laboratory. The system detects subtle changes in container vibration characteristics caused by differences in the physical properties of the fill materials. A container is inspected by acoustically inducing it to vibrate and sensing the vibrational response with a laser vibrometer. A standoff distance of several meters is feasible. In previous work the system proved to be a reliable means of distinguishing between munitions with a variety of chemical fills. During FY-93, the system was modified to improve performance and simplify operation. Other FY-93 accomplishments include progress in modeling the vibrational characteristics of containers and refinements to the statistical classification algorithms. Progress was also made in identifying other applications for this technology. 


\section{CONTENTS}

ABSTRACT $\ldots \ldots \ldots \ldots \ldots \ldots \ldots \ldots \ldots \ldots \ldots \ldots \ldots \ldots \ldots \ldots \ldots \ldots \ldots \ldots \ldots \ldots \ldots \ldots \ldots \ldots \ldots \ldots \ldots$
INTRODUCTION $\ldots \ldots \ldots \ldots \ldots \ldots$

DESCRIPTION OF LASER-ACOUSTIC INSPECTION $\ldots \ldots \ldots \ldots \ldots \ldots \ldots \ldots$

SOFTWARE DEVELOPMENTS $\ldots \ldots \ldots \ldots \ldots \ldots \ldots \ldots \ldots \ldots \ldots \ldots$

HARDWARE UPGRADES $\ldots \ldots \ldots \ldots \ldots \ldots \ldots \ldots \ldots \ldots \ldots \ldots \ldots \ldots$

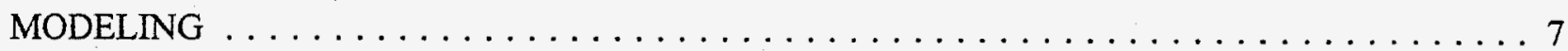

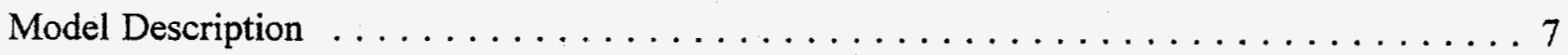

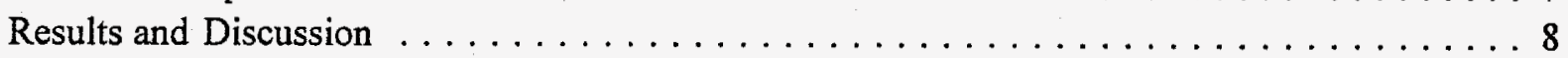

Finite Element Validation $\ldots \ldots \ldots \ldots \ldots \ldots \ldots \ldots \ldots \ldots \ldots \ldots \ldots \ldots$

DETAILED STATISTICAL ANALYSIS EXAMPLE $\ldots \ldots \ldots \ldots \ldots \ldots \ldots \ldots$

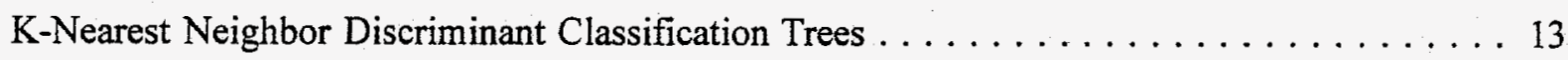

Discrimination Using the $\mathrm{KNN}$ Classification Tree Method . . . . . . . . . . . . . . 14

Example: Classification of Fill Types for $155 \mathrm{~mm}$ Artillery Shells $\ldots \ldots \ldots \ldots \ldots$

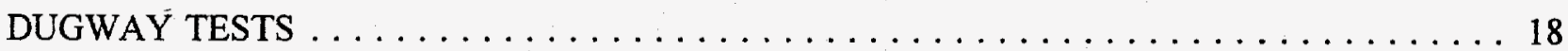

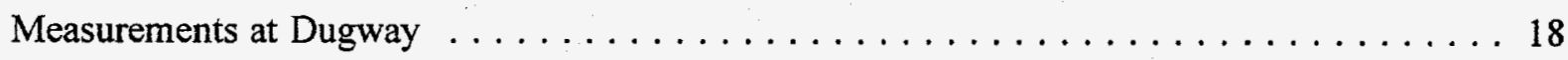

Analysis of Data from Muddy and Clean $155 \mathrm{~mm}$ Shells . . . . . . . . . . . . . 19

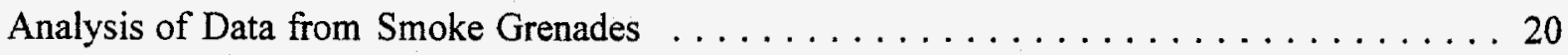

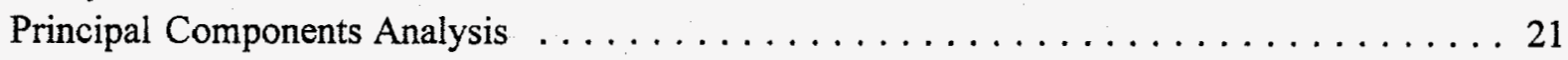

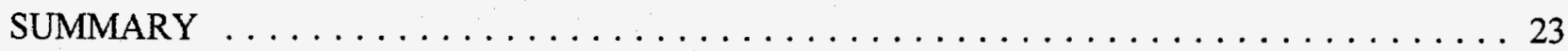

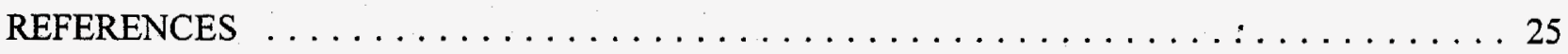

\section{Figures}

1. The effects of different fill types on resonance spectra of $155 \mathrm{~mm}$ artillery shells. . . . . . 2

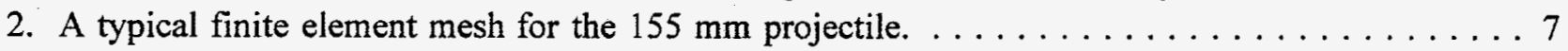

3. Vibrational modes for a hollow cylinder. $\ldots \ldots \ldots \ldots \ldots \ldots \ldots \ldots \ldots \ldots \ldots \ldots$

4. Typical computed mode shapes for the $155 \mathrm{~mm}$ projectile. . . . . . . . . . . . 9

5. Comparison of the computed natural frequencies for empty and paraffin-filled projectiles. . . . 9

6. Examples of natural frequencies specific to the internal burst tube. $\ldots \ldots \ldots \ldots \ldots \ldots$

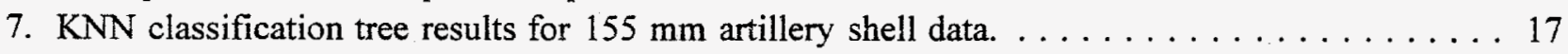

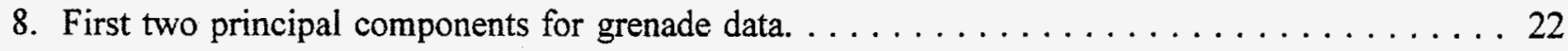




\section{Tables}

1. Parameters specified by the operator. . . . . . . . . . . . . . . . . 4

2. Computed natural frequencies for an empty $155 \mathrm{~mm}$ projectile. $\ldots \ldots \ldots \ldots \ldots$

3. Comparison of computed natural frequencies for empty and paraffin-filled $155 \mathrm{~mm}$

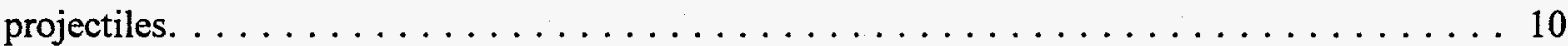

4. Computed natural frequencies for an actual $155 \mathrm{~mm}$ projectile, both without and with an internal burst

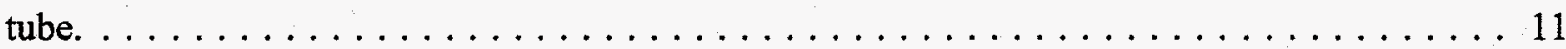

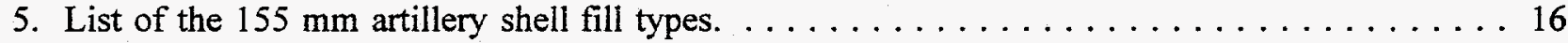

6. Classification tree global sensitivity results. $\ldots \ldots \ldots \ldots \ldots \ldots \ldots \ldots \ldots \ldots \ldots \ldots$

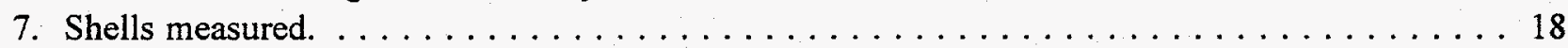

8. Grenade types measured. . . . . . . . . . . . . . . . . . . . . . . . 19

9. Comparison of mean frequency and amplitude values for muddy vs. clean $155 \mathrm{~mm}$ shells. . . . . . . . . . . . . . . . . . . . . . . . . . . . . . . 20 


\section{FY-93 Noncontacting Acoustic Ultrasonic Signature Analysis Development}

\section{INTRODUCTION}

The goal of this project has been to develop a practical and reliable noncontacting inspection system for containers. With funding from the U.S. Department of Energy, Office of Nonproliferation and National Security, work began in FY-91 to develop methods to aid in verification of chemical treaty compliance. Methods were developed for identifying the chemical contents of munitions and chemical agent storage containers. A portable inspection system was developed and demonstrated during field trials at the Tooele Army Depot in FY-91 and again in FY-92.

In FY-93, the focus of development efforts was broadened to include weapons inspection scenarios beyond chemical treaty verification, such as munition inspection for chemical munition destruction facilities. Nonmilitary applications, e.g., the inspection of food containers and hazardous waste containers, were also considered.

Enhancements to the inspection system in FY-93 were primarily geared toward making the system easier to use. An improved user interface to the controlling computer, utilizing graphical windows, was developed to simplify operation. The acquisition of a new fiber-less optical head has also simplified operation while improving the quality of measured data.

Modeling, initiated in FY-92, continued in FY-93. The goals of this effort are to improve data acquisition procedures and data analysis. Although the effort has so far concentrated on $155 \mathrm{~mm}$ artillery shells, extending the models to other cylinder-like containers is straightforward. Finite element analysis identified the frequencies and spatial characteristics of resonance modes of artillery shells. Some results were verified by laboratory experiments. This work has advanced to the point that the models are beginning to predict optimum data acquisition procedures.

Other accomplishments of FY-93 include modifications to the electronics to simplify the system, make it more compact, and increase the data collection speed. The FY-93 accomplishments summarized here are described in more detail in the body of the report. 


\section{DESCRIPTION OF LASER-ACOUSTIC INSPECTION}

The laser-acoustic inspection system operates on the principle that fill material affects the vibration of the container in a measurable and predictable way. Typically, the density, viscosity, and elastic properties of the fill material affect the frequencies at which vibration resonances occur and the amplitudes of those resonances. Figure 1 illustrates the effects of different fills on the resonance modes of a $155 \mathrm{~mm}$ artillery shell. The liquid-filled shell exhibits resonances that are shifted downward in frequency relative to the corresponding resonances in the empty shell. Also, the fraction of the total energy that is distributed in the higher frequencies is lower for liquid-filled shells than it is for empty shells. Clearly, the solid fill attenuates resonances above $3 \mathrm{kHz}$ much more than the other fills.

Vibrations are induced in objects by broadband white noise emanating from an ordinary loudspeaker. The loudspeaker is usually positioned as close to the inspected object as is practical. This distance is typically several inches, but has been as far as several feet.

The response of objects to acoustic excitation is sensed using a noncontacting laser vibrometer manufactured by Dantec Electronics. The vibrometer focuses approximately $2.5 \mathrm{~mW}$ of laser light on the surface of the object. The surface motion of the excited object induces a Doppler shift in the retroreflected laser light, which is sensed at a photodiode. Electronic demodulation of the Doppler-shifted light produces a voltage at the analog output of the sensor that is proportional to the instantaneous surface velocity. This output is lowpass filtered, to prevent aliasing, and then digitized.

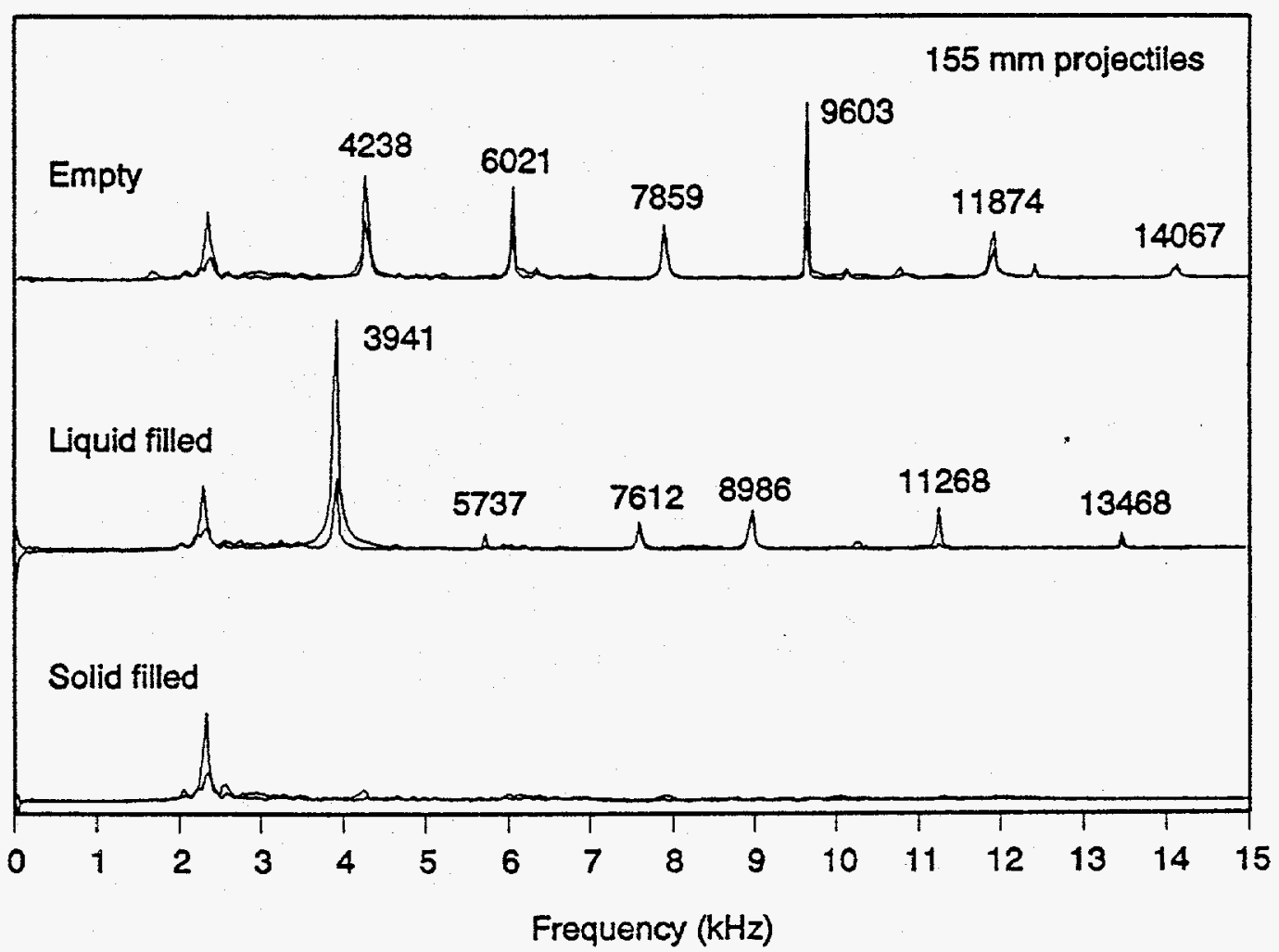

Figure 1. The effects of different fill types on resonance spectra of $155 \mathrm{~mm}$ artillery shells. 
Spectra are estimated from the digital data by averaging fast Fourier transforms (FFTs). Typically, spectra are estimated by averaging 200 FFTs. Each FFT is typically computed from 2048 data points, collected at a sampling rate of $50 \mathrm{kHz}$ or $83.3 \mathrm{kHz}$.

Classification of an inspected object is accomplished by statistical processing of the object's frequency spectrum. The statistical technique employed is a nonparametric "k nearest neighbor" method, where the value of $k$ is usually 3. A set of spectra from objects of known class is required before an unknown spectrum can be categorized. Classification is based on the "proximity" of the spectrum of the unknown object to known spectra.

The input to the classification algorithm is a set of frequency and amplitude pairs. Each pair is the frequency and amplitude of the largest resonance peak in a specific frequency range of the calculated spectrum. Typically, 16 nonoverlapping frequency ranges will be used, resulting in a total of 32 parameters characterizing each spectrum. Each set of 32 parameters defines a point in parameter space. That point will be classified based on its proximity to other points of known class. An item of unknown class is assigned to a class based upon the class membership of its three nearest neighbors. If two of the three nearest neighbors belong to the same class, then the unknown item will be assigned to that class. The determination of "proximity" is based on the Mahalanobis distance between points:

$$
d(\mathbf{x}, \mathbf{y})=\sqrt{(\mathbf{x}-\mathbf{y})^{\prime} \mathbf{V}^{-1}(\mathbf{x}-\mathbf{y})}
$$

The Mahalanobis distance is similar to the Euclidean distance, except that it is "adjusted" based on the intercorrelations of the variables in the study. The Mahalanobis distance to a point of a known class will be smaller if the point belongs to a broadly scattered class of points than if it belongs to a tightly grouped class of points.

The statistical analysis of collected spectra is currently done in the laboratory, not at the time of acquisition, because some preprocessing of the data is usually required. This situation will change as the statistical analysis is integrated into the data acquisition process. Eventually, the system will automatically do the classification analysis immediately after data acquisition. 


\section{SOFTWARE DEVELOPMENTS}

The parameters that the operator currently must specify to initiate data acquisition are described in Table 1. A graphical user interface was developed to facilitate the entry of these parameters. The operator is presented with a "form", which retains values from previous executions. The operator merely has to edit the form and then execute it. Often the only field that will require editing will be the name of the disk file containing the frequency spectrum. After data acquisition, the spectrum estimation program returns to the form.

Once initiated by the operator, data acquisition proceeds under computer control until a spectrum estimate has been acquired. A typical data acquisition sequence includes the following steps, performed by the computer:

1. Turn on the loudspeaker.

2. Compute a frequency spectrum by averaging 100 FFTs. Each FFT is computed from 2048 data points sampled at a rate of $83.3 \mathrm{kHz}$.

3. Write the frequency spectrum to disk.

Table 1. Parameters specified by the operator.

\begin{tabular}{lc}
\multicolumn{1}{c}{ Parameter description } & Typical value \\
\hline Number of points in each FFT record & 2048 \\
Number of FFTs averaged & 100 \\
Sample rate & $83 \mathrm{kHz}$ \\
Signal highpass filter cutoff & $50 \mathrm{~Hz}$ \\
Signal lowpass filter cutoff & $20 \mathrm{kHz}$ \\
Signal gain & 1 \\
White noise gain & .1 \\
White noise lowpass filter cutoff & $10 \mathrm{~Hz}$ \\
White noise highpass filter cutoff & $20 \mathrm{kHz}$ \\
Spectra estimation window definition & Hamming \\
Output file name & \\
Data set label & \\
\hline
\end{tabular}


4. With the loudspeaker still on, compute a second frequency spectrum by averaging $100 \mathrm{FFTs}$; write the spectrum to disk.

5. Turn the loudspeaker off.

6. Compute a frequency spectrum by averaging 100 FFTs, and write the spectrum to disk.

7. Repeat step 6.

8. Average the first two spectra together (collected with the loudspeaker on) and subtract the average of the last two spectra (collected with the loudspeaker off). Write the difference to disk.

An important step was taken in establishing a software configuration management plan. The software is now extensive enough that configuration management is necessary. The management plan details the procedures for tracking software development and managing changes. 


\section{HARDWARE UPGRADES}

The essential hardware components of the laser-acoustic inspection system are as follows:

1. Laser vibrometer

2. Controlling computer

3. Data acquisition board for sampling the vibrometer output

4. Analog filters and instrument amplifier for preprocessing the vibrometer output signal

5. Function generator for generating white noise

6. Audio amplifier for amplifying the white noise

7. Loudspeaker for broadcasting the white noise excitation.

The controlling computer has an ISA bus and an 80386-compatible processor. The data acquisition board is located in an expansion slot inside the computer case.

In FY-93, a circuit board for the preprocessing electronics and function generator was built and installed in an expansion slot in the computer and a small, $60 \mathrm{~W}$ audio amplifier was mounted inside the computer case. As a result of these improvements, the inspection system is more compact and has fewer components. Data acquisition proceeds a little faster also, because the new white noise generator is initialized faster than the previous one. All preprocessing and noise generating functions are now under software control. This is advantageous because nearly all the parameters associated with data collection are now available to the software, and can be stored with the estimated spectra. This relieves the operator of the task of documenting these parameters.

Another important system upgrade achieved in FY-93 was the acquisition of a non-fiber optical head for the laser vibrometer system. The old optical head used an optical fiber, approximately 10 feet in length, to receive light from the laser. The orientation of the fiber affected the polarization of the transmitted light, which affected vibrometer performance. The laser is now connected directly to the new optical head, eliminating the need for a long fiber. The new fiber-less system is measurably easier to use and not as susceptible to random noise as the fiber-based sensor.

The hardware modifications described here are part of the overall plan to develop a mobile, high performance, user friendly inspection system. 


\section{MODELING}

A finite element numerical model of the $155 \mathrm{~mm}$ projectile was constructed to improve understanding of the projectile vibrational response and to assist in determining feasibility of the overall project. Specific modeling goals included: (1) estimation of the effect of projectile contents (e.g., empty, fluid, solid) on natural frequencies, (2) prediction of optimal experimental measurement locations on the projectile surface, and (3) estimation of the effect of geometrical variances (e.g., due to manufacturing tolerances) on the projectile vibrational response.

\section{Model Description}

To simplify the analysis, the projectile was initially assumed to be unfilled and have no internal burst tube. Although the projectile is naturally axisymmetric, a fully three-dimensional model was constructed in order to capture both symmetric and asymmetric vibrational modes. Geometric details were extracted from rough drawings using graphical approximations where dimensioning details were inadequate. Using the PATRAN ${ }^{1}$ mesh generator, a variety of computational meshes were created from three-dimensional finite elements (20-node bricks); a typical mesh is shown in Figure 2. Then, with interface software, the PATRAN generated meshes were translated into node and element input data for the ABAQUS ${ }^{2}$ finite element software package. To study the effects of small geometry variations due to manufacturing tolerances, for one calculation the projectile exterior wall thickness was increased by $10 \%$. For a second calculation, the projectile was filled with a solid material (paraffin).

For later simulations, actual wall thickness measurements (taken from the device examined experimentally) were employed to provide a more accurate description of the projectile geometry. Additional geometric details, ignored for reasons of simplicity in the first series of calculations, were also included. In one case, the internal steel burst tube was included to study its effects on the projectile response. For this revised geometry, only empty projectiles were considered. In all simulations, both the steel projectile and paraffin filler were assumed isotropic with the following elastic properties and density:

$\begin{array}{lcc} & \text { Steel } & \text { Paraffin } \\ \text { Young's Modulus } & 207 \mathrm{GPa} & 1.7 \mathrm{GPa} \\ \text { Poisson's Ratio } & 0.292 & 0.45 \\ \text { Density } & 7861 \mathrm{~kg} / \mathrm{m}^{3} & 900 \mathrm{~kg} / \mathrm{m}^{3}\end{array}$

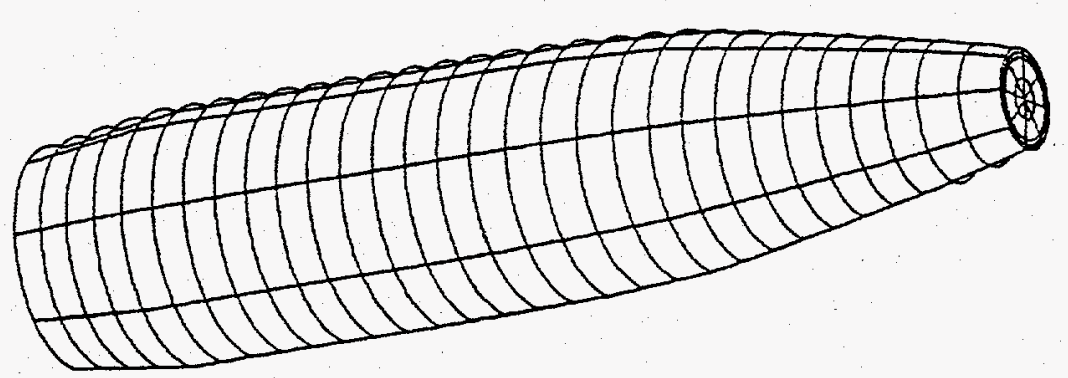

Figure 2. A typical finite element mesh for the $155 \mathrm{~mm}$ projectile. 
ABAQUS computes natural frequencies using the subspace iteration technique, which is a linear perturbation procedure. The reader is referred to the ABAQUS documentation ${ }^{2}$ for additional details.

\section{Results and Discussion}

The finite element eigenvalue extraction analysis provides output data in two forms. First, a list of eigenvalues and natural frequencies is provided, ordered from lowest to highest frequency. The extent of this list is controlled by user input (typically only the lower frequencies are of interest). Secondly, a plot of the vibrational mode shape is available at each frequency. In all cases, the projectile displayed nodal patterns similar to those of a hollow cylinder. Figure 3 shows the expected circumferential nodal

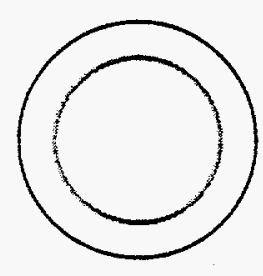

$n=0$

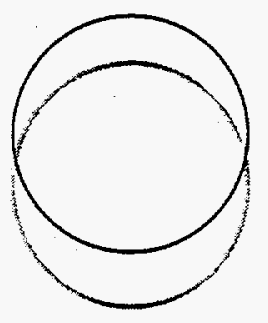

$n=1$

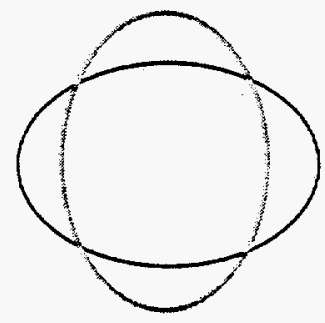

$n=2$

\section{CIRCUMFERENTIAL NODAL PATTERN}

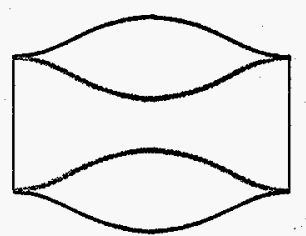

$m=1$

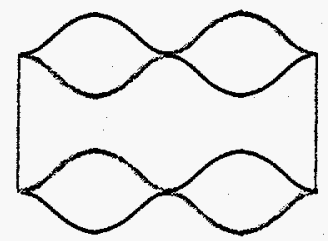

$m=2$

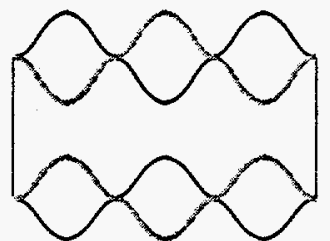

$\mathbf{m}=\mathbf{3}$

AXIAL NODAL PATTERN

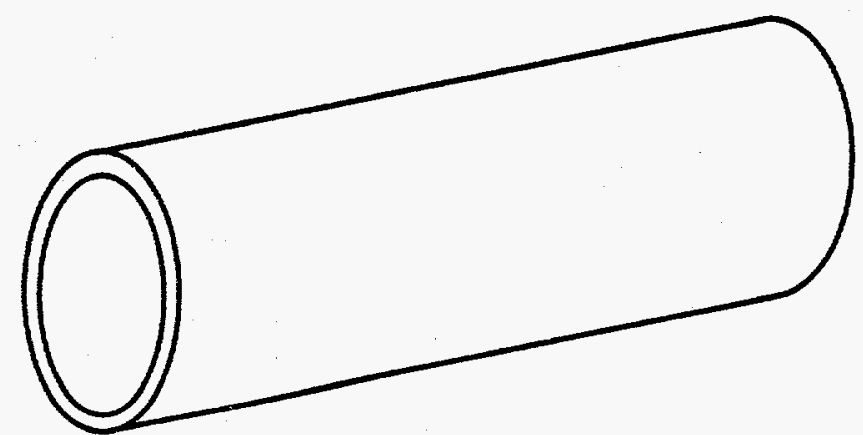

Figure 3. Vibrational modes for a hollow cylinder. 
patterns (identified as $n=0,1,2, \ldots$ ) and axial nodal patterns (identified as $m=1,2, \ldots$ ) for a vibrating cylinder. ${ }^{3}$ The nomenclature of this figure will be used to describe the projectile natural frequency results.

The numerical analysis provides a greater number of natural frequencies than are observed using the experimental technique described previously. This is because the experimental apparatus is only sensitive to out-of-plane displacements, thus only modes exhibiting significant out-of-plane motion are observed. Consequently, the first circumferential mode $(n=0)$, or axial plunging, typically is not evident in the experimental results. Judging by a relatively close frequency match between experimental and simulation results, two-lobe circumferential ringing $(n=2)$ appears to be the mode to which the experimental technique is most sensitive. Typical computed mode shapes for the $155 \mathrm{~mm}$ projectile are shown in Figure 4. The first three axial modes $(\mathrm{m}=1,2,3)$ are displayed for two-lobe circumferential $(\mathrm{n}=2)$ mode vibration.

For the simplest geometry considered (no internal burst tube or filler material), the computed natural frequencies and corresponding modes are shown in Table 2. Also shown are results for a similar analysis wherein the projectile outer wall thickness was increased by $10 \%$. For the vibrational modes expected to be most significant in this study $(\mathrm{n}=2 ; \mathrm{m}=1,2,3, \ldots)$, increasing the wall thickness increases the vibration frequency by approximately 3 to $5 \%$. The first two circumferential modes $(n=0,1)$ are significantly less affected by wall thickness.

Table 3, similar to Table 2, compares natural frequency values for an empty and a filled projectile. For the filled case, the projectile is assumed to be completely filled with paraffin, i.e., there is no burst tube. For the two-lobe ringing mode $(n=2)$, the largest difference between the filled and empty projectiles is at the first axial mode $(\mathrm{m}=1)$ and is approximately $12 \%$. The two-lobe $(\mathrm{n}=2)$ results are plotted in Figure 5, along with some similar numerical results for a smaller $105 \mathrm{~mm}$ projectile analyzed at 2-lobe ringing $(n=2)$ Los Alamos National Laboratory. ${ }^{4}$ For both

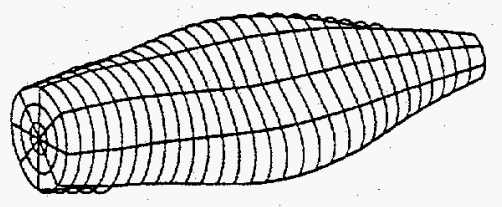

$2866 \mathrm{~Hz}$
$m=1$
$4050 \mathrm{~Hz}$
$m=2$


$5410 \mathrm{~Hz}$
$m=3$
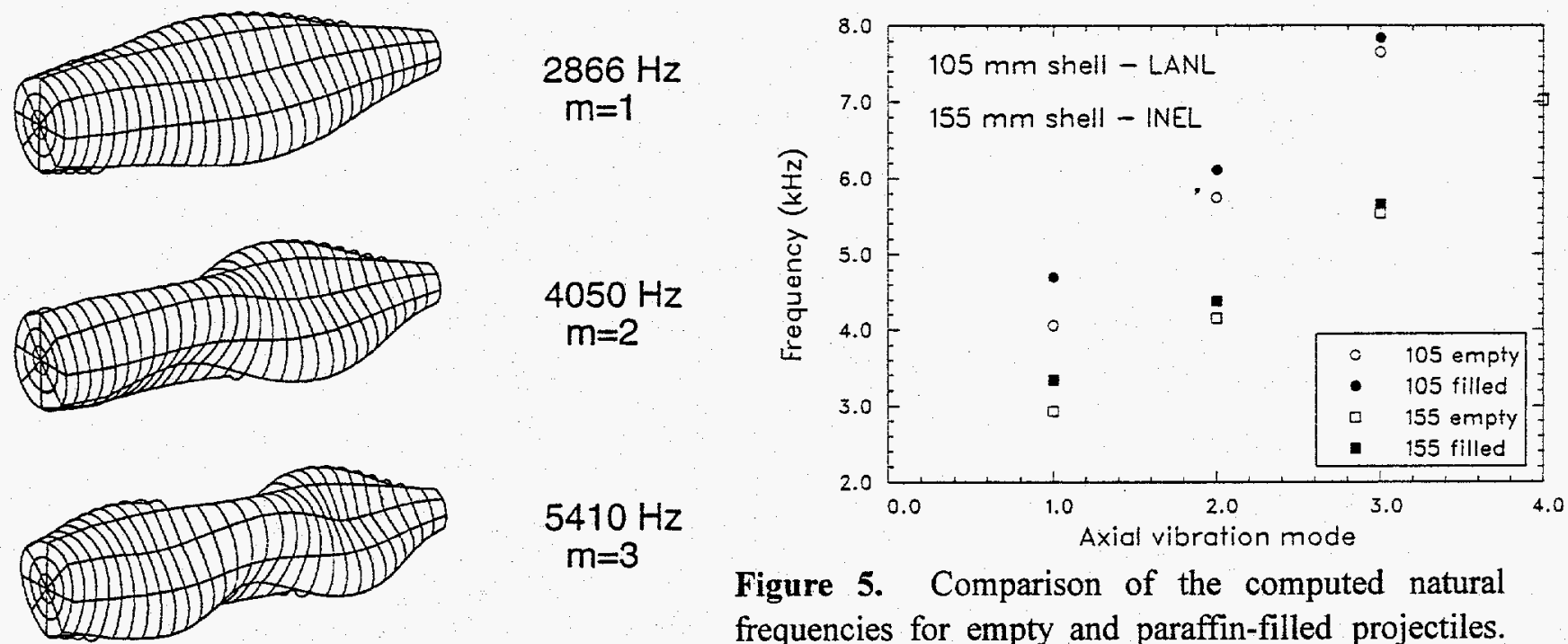

Figure 5. Comparison of the computed natural frequencies for empty and paraffin-filled projectiles.

Figure 4. Typical computed mode shapes for the $155 \mathrm{~mm}$ projectile. Displacements are highly magnified. The plotted values are for a two-lobe circumferential mode vibration. The LANL data are extracted from Reference 4. 
Table 2. Computed natural frequencies for an empty $155 \mathrm{~mm}$ projectile.

\begin{tabular}{|c|c|c|c|}
\hline \multirow[b]{2}{*}{ Circumferential Mode, $\mathrm{n}$} & \multirow[b]{2}{*}{ Axial Mode, $\mathrm{m}$} & \multicolumn{2}{|c|}{ Natural Frequency (Hz) } \\
\hline & & Standard Thickness & $10 \%$ Increased Thickness \\
\hline \multirow[t]{2}{*}{0} & 1 & 4009 & 4034 \\
\hline & 2 & 7900 & 7897 \\
\hline \multirow[t]{3}{*}{1} & 1 & 1926 & 1953 \\
\hline & 2 & 3748 & 3789 \\
\hline & 3 & 5666 & 5708 \\
\hline \multirow[t]{3}{*}{2} & 1 & 2866 & 3003 \\
\hline & 2 & 4050 & 4200 \\
\hline & 3 & 5410 & 5583 \\
\hline \multirow[t]{2}{*}{3} & 1 & 7024 & 7389 \\
\hline & 2 & 8446 & 8496 \\
\hline
\end{tabular}

Table 3. Comparison of computed natural frequencies for empty and paraffinfilled $155 \mathrm{~mm}$ projectiles.

\begin{tabular}{cccc}
\hline & & \multicolumn{2}{c}{ Natural Frequency $(\mathrm{Hz})$} \\
\cline { 3 - 4 } Circumferential Mode, $\mathrm{n}$ & Axial Mode, $\mathrm{m}$ & Empty & Paraffin-filled \\
\hline 0 & 1 & 4134 & 3982 \\
& 2 & 8105 & 5982 \\
1 & 1 & 1985 & 1918 \\
& 2 & 3851 & 3704 \\
& 3 & 5817 & 5528 \\
& 1 & 2931 & 3333 \\
& 2 & 4145 & 4380 \\
& 3 & 5534 & 5655 \\
& 1 & 6971 & 7184 \\
& 2 & 8443 & 8551 \\
& & & \\
\hline
\end{tabular}

projectiles, the frequency differences resulting from the solid filler are largest at the lowest axial vibration mode and diminish somewhat at higher frequencies. In comparing Tables 2 and 3 , note that small frequency differences are observed for the empty projectile in the two cases because slightly different (geometrically) empty projectiles were used in the two cases. 
As mentioned above, after an initial calculation and series of parametric studies, the model was refined to more closely represent the geometry of an actual projectile. Simulations were made both with and without the internal burst tube. The natural frequency results from these calculations are summarized in Table 4. Refining the geometry had a significant effect on the computed natural frequencies for all vibrational modes (see results for empty projectiles in Tables 2 through 4). Including the burst tube had a much smaller effect, particularly for the modes of most interest to this study $(n=2 ; m=1,2, \ldots)$. As shown in Figure 6, new natural frequencies specific to the burst tube are observed. However, the effect on the overall projectile response (particularly the outer surface where experimental data is collected) is minimal.

\section{Finite Element Validation}

For most experiments in this study, surface displacement data were taken at only a few locations on the projectile, making it impossible to determine vibrational modes. In one experiment, however, a much more complete data set was developed. In this case, data were obtained at seven axial and eight circumferential positions, providing data at 56 separate locations. This data set was sufficient to construct rough plots of both the axial and circumferential surface displacements and thus infer the vibrational mode.

Two experimentally determined frequencies were selected for comparison to the simulations. These corresponded to two frequencies that have been observed in all prior experiments and for which the displacements were largest. From the rough displacement plots described above, it was evident that the lowest frequency, $2766 \mathrm{~Hz}$, corresponded to the first axial mode $(\mathrm{m}=1)$ of a two-lobe circumferential $(\mathrm{n}=2)$ mode vibration. Likewise, the second frequency, $3876 \mathrm{~Hz}$, was the second axial mode $(\mathrm{m}=2)$ of the two-lobe vibration. The computed frequencies for these two modes, 2660 and $3748 \mathrm{~Hz}$, respectively, are both within $4 \%$ of the experimental value. This excellent agreement between numerical

Table 4. Computed natural frequencies for an actual $155 \mathrm{~mm}$ projectile, both without and with an internal burst tube.

\begin{tabular}{cccc} 
& & \multicolumn{2}{c}{$\begin{array}{c}\text { Natural Frequency } \\
(\mathrm{Hz})\end{array}$} \\
\cline { 3 - 4 } $\begin{array}{c}\text { Circumferential } \\
\text { Mode, } \mathrm{n}\end{array}$ & $\begin{array}{c}\text { Axial } \\
\text { Mode, } \mathrm{m}\end{array}$ & $\begin{array}{c}\text { Without } \\
\text { Burst } \\
\text { Tube }\end{array}$ & $\begin{array}{c}\text { With } \\
\text { Burst } \\
\text { Tube }\end{array}$ \\
\hline 0 & 1 & 4356 & 4226 \\
1 & 1 & 2102 & 2026 \\
& 2 & 4069 & 4030 \\
2 & 1 & 2647 & 2660 \\
& 2 & 3734 & 3748 \\
& 3 & 4940 & 4966
\end{tabular}


analysis and experiment provides added confidence in the simulations.

A primary goal of the modeling effort is to identify the appropriate number of measurements and optimal locations for those measurements on the surface of the inspected object. To accomplish this gcal, information is needed on the shape, frequency, and amplitude of the resonance modes and on how variations in fill material affect those characteristics. In addition, it is necessary to know the sensitivity of each resonance mode to minor deviations in geometry and material properties. With this information it should be possible to identify the most useful resonance modes for the inspection task at hand and devise a measurement strategy to sample those resonance modes in an optimal fashion. The numerical results described here indicate that the modeling effort is approaching the level of sophistication necessary to achieve this goal. 


\section{DETAILED STATISTICAL ANALYSIS EXAMPLE}

As mentioned earlier, classification of spectra is accomplished using a nonparametric k-nearest neighbor $(\mathrm{KNN})$ discriminant analysis technique. Because $\mathrm{KNN}$ discriminant analysis tends to deteriorate as the number of classes increases, a classification method based on a combination of KNN with classification tree techniques was developed. Key features of the method are that it allows (1) a readily constructed and interpretable tree based on natural hierarchies among the classes, (2) classification into more than two groups at each step when appropriate (i.e. when the natural classification hierarchy suggests it), and (3) multiple criteria at each step. Definition of the classification tree is, of course, very application dependent. A detailed example of the classification method, applied to the problem of identifying chemical weapon fill types, is described here.

\section{K-Nearest Neighbor Discriminant Classification Trees}

Standard methods of discriminant analysis (e.g. linear discriminant analysis, quadratic discriminant analysis) require that the data follow a normal (Gaussian) distribution. The accuracy of these methods suffers when this requirement is not met. Because acoustical spectra data generally do not follow a known distribution, we use the nonparametric k-nearest neighbor method of discriminant analysis as the basis for classifying objects.

Formally, k-nearest neighbor discriminant analysis works as follows. Suppose an object is known to belong to one of a set of $q$ mutually exclusive classes $C=\left\{c_{1}, c_{2}, \ldots, c_{q}\right\}$, and we wish to assign the object to one of these classes based on $\mathbf{x}$, a vector of measured characteristics of that object. Given a set of training data, the analysis must assign the object to class $\mathrm{j}$ such that

$$
\frac{\mathrm{k}_{\mathrm{j}} / \mathrm{n}_{\mathrm{j}}}{\mathrm{k}_{\mathrm{i}} / \mathrm{n}_{\mathrm{i}}}>\pi_{\mathrm{i}} / \pi_{\mathrm{j}} \quad \text { for all } \mathrm{i} \neq \mathrm{j}
$$

where $k_{i}$ is the number of the k-nearest neighbors to $x$ that are in class $i, n_{j}$ is the number of members of class $i$ in the training set, and $\pi_{i}$ is the population proportion in class $i$ (or some other estimate of the prior probability of membership in class i). Choosing the value of $k$ is rather arbitrary (typically between 3 and 5) and will not have much effect on correct classification rates in a well-behaved classification system. We generally use $\mathrm{k}=3$.

Determining which points in the training set are the k-nearest neighbors to $\mathbf{x}$ requires specifying a distance metric. If the true covariances among elements of $\mathbf{x}$ are known, then the probability of correct classification is maximized by using the Mahalanobis distance with the known class-specific covariance matrix. The Mahalanobis distance between $\mathbf{x}$ and an object $\mathbf{x}_{\mathrm{i}}$ known to belong to class $\mathrm{i}$ is

$$
d\left(\mathbf{x}, \mathbf{x}_{\mathbf{i}}\right)=\left(\left(\mathbf{x}-\mathbf{x}_{\mathbf{i}}\right)^{\prime} \mathbf{V}_{\mathbf{i}}^{-1}\left(\mathbf{x}-\mathbf{x}_{\mathbf{i}}\right)\right)^{1 / 2}
$$

where $V_{i}$ is the covariance matrix for class $i$. 
In practice, the covariance matrices are not known and must be estimated from the data on training objects, usually after making some assumptions regarding the form of the matrix. Generally, a common covariance structure across classes is assumed, and a pooled estimate is obtained. Thus, while KNN analysis does not require assumptions regarding probability distributions or estimation of mean vectors as do other discrimination methods, it does depend on assumptions regarding the covariance matrix structure and on the estimation of covariance terms.

As classes increase in number, the usual $\mathrm{KNN}$ assumption of equal covariance matrices becomes less tenable, suggesting that class-specific rather than pooled covariance matrix estimates should be used. But going to class-specific covariance matrices increases the number of parameter estimates required relative to the number of training objects available. Rarely are there enough data to provide adequate estimates of class-specific matrices. One solution to this problem is to combine $\mathrm{KNN}$ analysis with a classification tree approach.

In the broadest sense of the term, classification tree methods are simply any recursive partitioning techniques that break a discrimination problem into a sequential and branching series of decisions. Each branching point or node in the tree produces partial classification by focusing on comparisons between key variables and/or classes. Complete classification is achieved when all objects have been processed through the appropriate intermediate classification steps to reach a terminal point or leaf in the tree.

Using a classification tree approach to break a $\mathrm{KNN}$ classification task into smaller segments can mitigate some of the problems inherent with using nonrecursive $\mathrm{KNN}$ analysis on data containing large numbers of classes and/or measurement variables. Keeping the number of classes small increases the likelihood that between-class covariance matrices will have similar structures, so that using a pooled variance estimate is a more valid approach. All else being equal, whenever a classification tree approach has beneficial effects on the precision of covariance matrix estimates, it will theoretically improve classification results compared to using nonrecursive KNN analysis.

\section{Discrimination Using the KNN Classification Tree Method}

Suppose as before that there is a set of $q$ mutually exclusive classes $C=\left\{c_{1}, c_{2}, \ldots, c_{q}\right\}$ into which we wish to classify objects and for which certain measurements are available from a set of training objects with known class memberships. Formally, the KNN classification tree method proceeds in the following manner:

1. From the elements of $C$, determine a set of $p$ mutually exclusive aggregate groups of classes $G_{1}=$ $\left\{g_{11}, g_{12}, \ldots, g_{1 p}\right\}$ such that $2 \leq p<q$ (i.e., each element of $G_{1}$ contains one or more of the elements in $C$ ) into which to partition the training set data. (Note: $p$ is typically small, say two or three. If $\mathrm{p}=\mathrm{q}$, then it is a nonrecursive $\mathrm{KNN}$ classification.)

2. Select a vector of measurements $\mathbf{x}_{\mathrm{G1}}$ from those available on the training objects that are capable of discriminating between the classes in $G_{1}$.

3. Perform $\mathrm{KNN}$ classification analysis on the training set data, and assign each object to an element of $G_{1}$ according to the results of the nearest neighbor calculations. 
4. Evaluate the partitioning's adequacy by cross-validation. If results are unacceptable, repeat steps 1 through 3, making appropriate adjustments in either $G_{1}$ or $x_{G 1}$.

5. For each element of $\mathrm{G}_{1}$ composed of more than one element of $\mathrm{C}$, repeat steps 1 through 4 . For example, if $g_{11}$ contains $r>1$ elements of $C$, form a new set of aggregate groups $G_{11}=\left\{g_{111}, g_{112}\right.$, $\ldots\}$, where the elements of $\mathrm{G}_{11}$ are formed from the elements of $\mathrm{C}$ previously composing $\mathrm{g}_{11}$. Any element of $G_{1}$ composed of a single element from $C$ comprises a terminal node or leaf in the classification tree and is not analyzed further.

6. Repeat steps 1-5 until all elements of $C$ appear as terminal nodes.

Within the constraint of choosing partitions that increase the probability of correct classification, the aggregate groups are generally formed along the lines of natural hierarchies among the elements of C. The iterative component of the tree development specified in steps 1 through 4 provides an empirical feedback approach to fine-tuning the classification tree that complements and serves as a check on the relevant (i.e., useful) partitions suggested by the natural hierarchies of the classes.

The performance of a KNN classification tree is best judged by classifying new objects (i.e., ones not in the training set). In lieu of such new data, we used cross-validation (or the "leave-one-out" method) of the KNN discriminant analyses performed at each branching node in the classification tree. This serves as a basis for measuring the local discriminatory power (i.e., how well discrimination works at each node). Cross-validation treats $n-1$ out of $n$ training observations as a training set to classify the one remaining object. This is done for each of the $\mathrm{n}$ training objects, with the number of correct and incorrect classifications recorded for each node. The two measures of interest are sensitivity and specificity. Sensitivity measures the probability that a node captures objects belonging to it. Specificity measures the probability that a node captures only its own true members. Discrimination capabilities across multiple branching nodes is evaluated by combining the appropriate node-specific cross-validation results. Of particular interest are the $q$ terminal node paths, which give estimates of the probability of correct classification into each class in $\mathrm{C}$ for objects processed through the entire tree. Another useful measure is the total sensitivity or nonerror rate for the tree, i.e. the combined percent of objects correctly classified.

\section{Example: Classification of Fill Types for $155 \mathrm{~mm}$ Artillery Shells}

During field trials in 1992, data on empty $155 \mathrm{~mm}$ artillery shells and shells containing four types of chemical weapons (white phosphorous, GB, VX, and $\mathrm{H}$ ), three chemical weapon simulants (GB-S, VX$\mathrm{S}$, and H-S), and two surrogate fill materials (sand and ethylene glycol) were collected: Measurements were obtained on a total of 109 shells. Table 5 gives the number of items with each type of fill.

Prior to the statistical discriminant analysis, the data were preprocessed in several ways. First, data were merged so that each test item was represented by only one spectrum. Merging was accomplished by computing the mean amplitude at each frequency from the amplitudes in the various spectra. Next, it was necessary to create data files suitable for use by the statistical analysis program. Each spectrum was first processed by a peak detection program, which identifies spectral peaks and suppresses high frequency background noise. The peak-detected spectra were then analyzed to identify the frequency and amplitude of the highest spectral peak in each of a set frequency ranges. Sixteen contiguous frequency ranges were used in processing the $155 \mathrm{~mm}$ artillery shell data. The first eight ranges were $500 \mathrm{~Hz}$ wide, covering the frequencies between $0 \mathrm{~Hz}$ and $4 \mathrm{kHz}$. The next eight ranges were $1 \mathrm{kHz}$ wide, covering the 
Table 5. List of the $155 \mathrm{~mm}$ artillery shell fill types.

\begin{tabular}{lclc}
\hline Fill Material & $\begin{array}{c}\text { Number } \\
\text { Inspected }\end{array}$ & Fill Material & $\begin{array}{c}\text { Number } \\
\text { Inspected }\end{array}$ \\
\hline VX & 16 & Mustard Simulant & 5 \\
GB & 16 & Ethylene Glycol & 8 \\
Mustard & 17 & Sand & 8 \\
VX Simulant & 5 & Empty & 7 \\
GB Simulant & 5 & White Phosphorus & 22 \\
\hline
\end{tabular}

frequencies between $4 \mathrm{kHz}$ and $12 \mathrm{kHz}$. For the statistical analysis, each spectrum was thus characterized by 32 parameters: 16 frequencies and 16 corresponding amplitudes. (In addition average amplitude was also used as an analysis variable for some nodes in the tree.)

Skewness, kurtosis, and normal probability plots were examined for each of the classification variables, and the Shapiro-Wilks test for normality was performed. Results indicated severe departures from normality for many of the variables. The same tests on the logarithms and rank transformations of the variables indicated no consistent improvements in fit. Thus, there was a strong indication that a nonparametric method such as $\mathrm{KNN}$ would be more appropriate than other discriminant methods for these data. To further smooth the data, the KNN analysis was performed on data ranks.

All KNN analysis and validation was performed using PROC DISCRIM in the SAS software system for personal computers. ${ }^{5}$ Analysis was based on $\mathrm{k}=3$ nearest neighbors in all cases. The classification tree selected for the chemical weapons data is shown in Figure 7, with information on the node-specific cross-validation results. Ratios listed in the figure are cross-validation estimates of sensitivity, and percentages are the probability of correct classification for objects belonging to the node. Table 6 gives global sensitivity results for each munition fill type (i.e., calculated for each of the 10 paths resulting in a terminal node).

KNN classification tree analysis is a useful method for classifying objects inspected with the laser acoustic system, as indicated by cross-validation results in the example. The cross-validation results are somewhat optimistic in that the classification of actual new objects (rather than reclassification of those in the training set) will likely result in somewhat lower correct classification rates. Still, the method shows great promise. Future efforts will be directed at actual classification of new objects, and further investigation of optimal data preprocessing and data reduction procedures. 


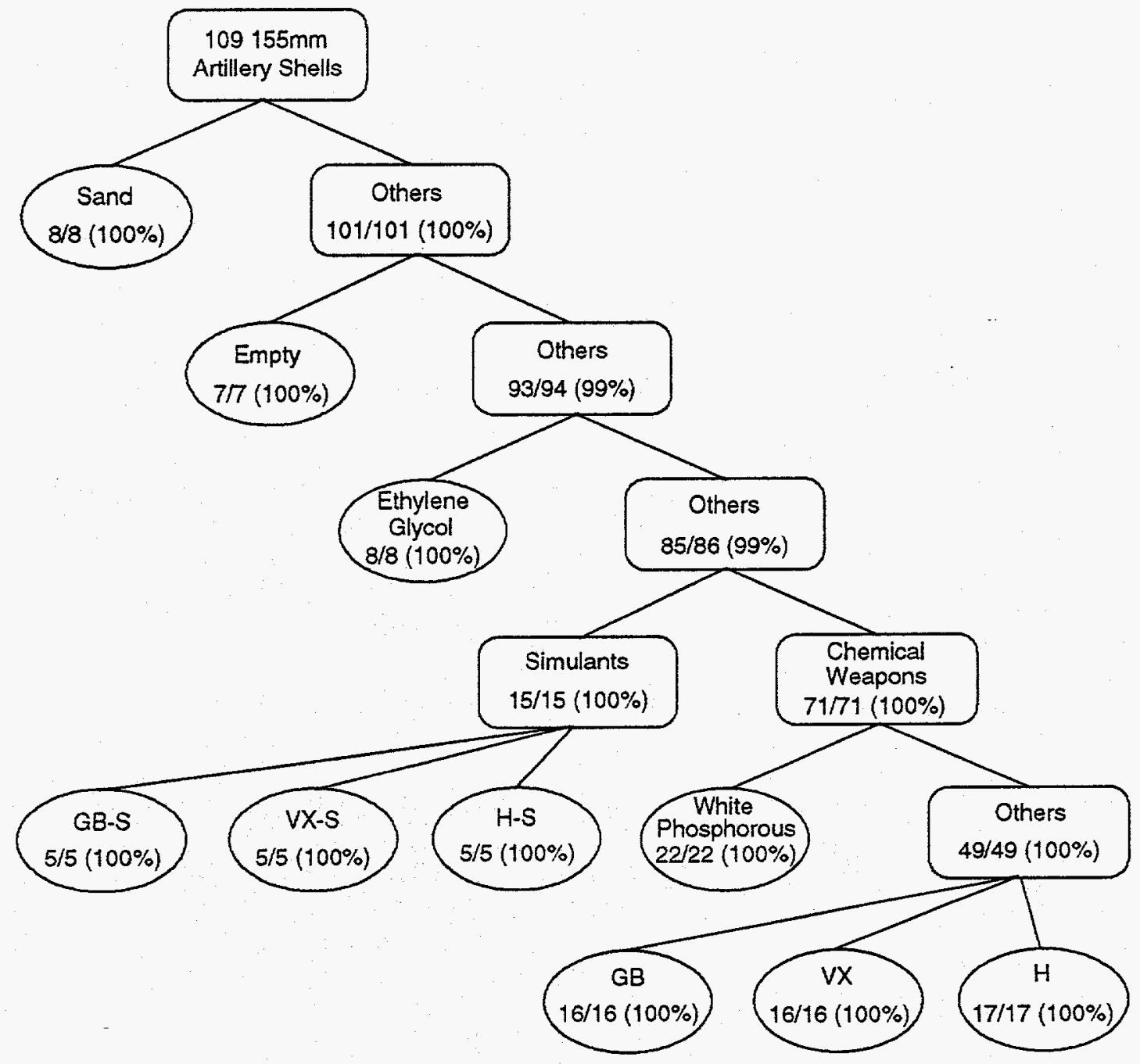

Figure 7. KNN classification tree results for $155 \mathrm{~mm}$ artillery shell data.

Table 6. Classification tree global sensitivity results.

\begin{tabular}{lccc}
\multicolumn{1}{c}{ Group } & Sensitivity (\%) & Group & Sensitivity (\%) \\
\hline Sand & 100 & H-S & 100 \\
Empty & 100 & White phosphorous & 100 \\
Ethylene glycol & 100 & GB & 100 \\
GB-S & 100 & VX & 94 \\
VX-S & 80 & H & 100 \\
& & & \\
\hline
\end{tabular}




\section{DUGWAY TESTS}

The Noncontacting Acoustic Ultrasonic Signature Analysis (NAUSA) system was tested at Dugway, Utah on April 19-20, 1993 to (1) determine its ability to obtain measurements from munition surfaces covered with mud, (2) determine the effect of mud on a munition signature, and (3) determine its ability to classify smoke grenades. This section describes the measurements taken and the statistical analysis performed.

\section{Measurements at Dugway}

During the two days of tests, the NAUSA system took more than 250 measurements on shells and grenades with and without mud. Measurements were taken for 21 different $155 \mathrm{~mm}$ munitions with 16 fill types and for 7 grenade types; the munitions measured are listed in Table 7, the grenade types in Table 8. The shells used (except for HC Smoke 155s) were configured without a burster tube. Also, due to the amount of material available for filling, these shells had various fill levels.

The first day, measurements were taken on clean $155 \mathrm{~mm}$ shells and grenades. Then the shells and $\mathrm{CN}$ grenades were randomly smeared with mud. The mud layer varied in amount and thickness over the shell's surface (in some cases the mud was so thick that it did not have time to dry completely); the

Table 7. Shells measured.

\begin{tabular}{|c|c|c|c|c|c|}
\hline $\begin{array}{l}\text { Shell } \\
\text { number }\end{array}$ & $\begin{array}{l}\text { Shell } \\
\text { weight }^{a} \\
\text { (lbs) }\end{array}$ & Identified fill & $\begin{array}{l}\text { Shell } \\
\text { number }\end{array}$ & $\begin{array}{l}\text { Shell } \\
\text { weight }^{\mathrm{a}} \\
\text { (lbs) }\end{array}$ & Identified fill \\
\hline 1. & 100.70 & Concrete & 12. & 96.10 & HC Smoke \\
\hline 2. & 92.80 & Dirt & 13. & 96.10 & HC Smoke \\
\hline 3. & 86.16 & Kerosene & 14. & 86.30 & Molasses \\
\hline 4. & 89.00 & Tin IV Chloride & 15. & 86.13 & Diesel Oil \\
\hline 5. & 86.10 & Carbon Tetrachloride & 16. & 86.10 & Empty \\
\hline 6. & 86.07 & Titanium Tetrachloride & 17. & 86.14 & Methyl Salicylate \\
\hline 7. & 86.10 & Empty & 18. & 86.17 & $\begin{array}{l}\text { Ethylene Glycol \& } \\
\text { Water }(50 / 50)\end{array}$ \\
\hline 8. & 86.10 & Empty & 19. & 86.08 & $\begin{array}{l}\text { Chloroform \& Water } \\
(25 / 75)\end{array}$ \\
\hline 9. & 96.10 & HC Smoke & 20. & 86.10 & Water \\
\hline 10. & 96.10 & HC Smoke & 21 & 86.10 & Gasoline \\
\hline 11. & 96.10 & HC Smoke & & & \\
\hline
\end{tabular}

a. Without mud. 
amount of mud placed on the shells was not measured. The locations of the measurement points on each munition relative to the test configuration were documented so that they would not be lost when mud was applied.

The NAUSA system took measurements directly on the mud surface, which posed no problems. Rather, data collection improved with the mud on the shell due to the greater signal-to-noise ratio. This ability to take vibrational information from muddy surfaces is an advantage over systems that require contact coupling for container identification.
Table 8. Grenade types measured.

\begin{tabular}{clc}
$\begin{array}{c}\text { Shell weight } \\
(\text { lbs })\end{array}$ & \multicolumn{1}{c}{ Identified fill } & $\begin{array}{c}\text { Number } \\
\text { measured }\end{array}$ \\
\hline 0.4939 & Violet Smoke (M18) & 10 \\
0.4446 & Green Smoke (M18) & 10 \\
0.5002 & Red Smoke (M18) & 2 \\
0.4383 & Yellow Smoke (M18) & 4 \\
0.8520 & TH (INCEN) & 13 \\
0.251 & CN (M6A1) & 16 (with and \\
& & without mud) \\
0.4807 & CS (M7A3) & 2 \\
\hline
\end{tabular}

\section{Analysis of Data from Muddy and Clean $155 \mathrm{~mm}$ Shells}

Acoustical spectra from the $21155 \mathrm{~mm}$ shells were analyzed using KNN discriminant analysis. Two spectra for each shell were obtained, one taken when the shell was clean and the other when it was covered with mud, for a total of 42 spectra. Each spectrum is a combination of data collected from two measurements on the shell. The spectra were adjusted for electronic noise and a peak detection routine was applied before the $\mathrm{KNN}$ analysis.

The frequency range of 0 to $12,000 \mathrm{~Hz}$ was used in the analysis. Data reduction was achieved by identifying the frequency and amplitude of the largest peak in specific frequency ranges. The frequency ranges considered are shown in Table 9. Intervals were concentrated in the lower frequency range to place more emphasis on the relatively more abundant peaks occurring in that range. The last two ranges contained no peaks for any of the spectra and were eliminated from the analysis. This yielded 28 variables for analysis (14 frequency and 14 amplitude variables).

The first KNN analysis was an attempt to pair up the two spectra (one muddy and one clean) for each shell. Since only one nearest neighbor of each spectrum can be its matching spectrum, the analysis was performed with $\mathrm{k}=1$ (i.e., classification is based on the single nearest neighbor). This analysis performed dismally. Only four pairs of muddy and clean spectra were correctly matched, showing the tendency for muddy and clean spectra to cluster separately. While this is not an impressive result, especially since $100 \%$ correct classification of whether or not a shell was muddy presumably could be achieved by visual inspection, it does show that muddy spectra look more like other muddy spectra than like clean spectra for the same shell. That is, mud on a shell apparently creates spectra characteristics that over-ride characteristics of the individual shell. When the muddy spectrum for a particular shell has nearest neighbors that are also muddy spectra, there is no possibility of matching it with the correct clean spectrum. 
Table 9. Comparison of mean frequency and amplitude values for muddy vs. clean $155 \mathrm{~mm}$ shells.

\begin{tabular}{|c|c|c|c|c|}
\hline \multirow{2}{*}{$\begin{array}{c}\text { Frequency Range } \\
\qquad(\mathrm{Hz})\end{array}$} & \multicolumn{2}{|c|}{$\begin{array}{l}\text { Mean Peak Frequency } \\
(\mathrm{Hz})\end{array}$} & \multicolumn{2}{|c|}{ Mean Peak Amplitude } \\
\hline & Clean & Mud & Clean & Mud \\
\hline $0-500$ & 164.6 & 186.2 & .061 & .060 \\
\hline $501-1000$ & $--^{a}$ & 739.9 & $\cdots$ & .009 \\
\hline $1001-1500$ & 1235.0 & 1240.6 & .026 & .020 \\
\hline $1501-2000$ & 1831.4 & 1834.0 & .769 & 1.029 \\
\hline $2001-2500$ & 2196.7 & 2195.8 & 2.51 & 2.44 \\
\hline $2501-3000$ & 2691.6 & 2681.0 & 20.8 & 12.1 \\
\hline $3001-3500$ & 3284.4 & 3292.2 & .302 & .479 \\
\hline $3501-4000$ & 3749.8 & 3787.5 & .325 & .769 \\
\hline $4001-5000$ & 4385.4 & 4367.7 & .628 & .124 \\
\hline $5001-6000$ & 5336.9 & 5478.8 & .283 & .004 \\
\hline $6001-7000$ & 6308.3 & 6272.6 & 8.22 & 1.04 \\
\hline $7001-8000$ & 7564.7 & 7519.1 & .014 & .041 \\
\hline $8001-9000$ & 8610.8 & 8598.9 & .012 & .003 \\
\hline $9001-10000$ & 9344.5 & 9396.5 & .039 & .005 \\
\hline $10001-11000$ & $-\cdots$ & - & $\cdots$ & -- \\
\hline $11001-12000$ & $\cdots$ & -- & $\cdots$ & -- \\
\hline
\end{tabular}

Overall, there were no consistent shifts in frequencies or changes in amplitudes between the muddy and clean spectra (see Table 9). Seven frequency values were smaller for clean spectra than for muddy spectra, while six were greater. Four amplitude values for clean spectra were smaller than for muddy spectra; 9 were larger. In the ranges with the 3 largest peaks $(2001-2500 \mathrm{~Hz}, 2501-3000 \mathrm{~Hz}$, and $6001-7000 \mathrm{~Hz}$ ), more consistent results are evident. In these three ranges, frequencies were all slightly higher for clean than muddy spectra, although differences were not great. However, there were some large differences in the amplitude values for these three peaks. Thus, mud on a shell seems to have a dampening effect on its spectrum.

\section{Analysis of Data from Smoke Grenades}

Acoustical frequency spectra from 10 violet, 10 green, and 4 yellow smoke grenades were analyzed using KNN discriminant analysis. Replicate spectra were available for each of the 4 yellow smoke grenades, so 28 spectra were analyzed. (Spectra from 2 red grenades were also available but not analyzed because two objects are not sufficient to define a group for analysis.) Other types of grenade fills (thermite, etc.) were not included because of differences in canister types.

The frequency range of 400 to $9600 \mathrm{~Hz}$ was used in the analysis. Two general analyses were performed, using two different methods of preliminary data reduction. Both analyses are based on identifying the frequency and amplitude of the largest peak in specific frequency ranges. In the first 
analysis, the frequency ranges used were (in Hz): 400-800, 800-1200, 1200-1600, 1600-2000, 2000-2400, $2400-4000,4000-6000,6000-8000$, and $8000-9600$. This yielded 18 variables for analysis ( 9 frequency and 9 amplitude variables). Intervals were concentrated in the lower frequency range to place more emphasis on the relatively more abundant peaks occurring in that region. The second analysis had increased focus on lower frequency peaks; the frequency range considered was reduced and narrower frequency bands within that range were used. Specifically, the frequency and amplitude of the largest peak in each $200 \mathrm{~Hz}$ interval in the range 400 to $2400 \mathrm{~Hz}$ were used, for a total of 20 variables.

In the first analysis, using 18 variables in a $\mathrm{KNN}$ discriminant analysis (with $\mathrm{k}=3$ nearest neighbors) resulted in two misclassifications among the 28 spectra (all classifications were based on cross-validation results). One green grenade spectrum was classified as yellow, and one yellow grenade was classified as green. This corresponds to correct classification rates of $90 \%, 100 \%$, and $88 \%$ for green, violet, and yellow grenades, respectively. The overall correct classification rate was $93 \%$.

The second analysis, which focused more on the lower frequency ranges, yielded better results. In that analysis, $100 \%$ correct classification of all spectra was obtained (again using $k=3$ ). Furthermore, all classification probabilities were 1.0, indicating less ambiguity in the classification procedure. (For example, a green grenade spectrum may have a 0.67 probability of membership in green, 0.33 in yellow, and 0.0 in violet, which is a less precise classification than 1.0 membership in green and 0.0 in yellow and violet.) Further analysis indicated that as few as 8 of the 20 frequency and amplitude variables were necessary to obtain $100 \%$ correct classification (although not all classification probabilities were 1.0 ).

To check for internal consistency of the data, the four sets of replicate spectra for the yellow smoke grenades were analyzed as a group to determine if the KNN method could correctly match the two spectra for each of the four grenades. Using the 18 variables from the first analysis above, all four pairs of spectra were correctly grouped together. (The value of $k$ was set equal to 1 in this $\mathrm{KNN}$ analysis since each spectrum had only one nearest neighbor that it could match correctly.)

\section{Principal Components Analysis}

The KNN analysis used 16 different frequency slices of the spectra collected for each container. These 16 slices incorporate frequency and amplitude information for the clustering algorithm used for material classification. A more robust way to replace this selected information without using all the information is provided by principal component analysis. Principal components are linear combinations of a set of variables (in this case frequency and amplitude values for the grenade acoustical signatures) that retain as much of the information contained in the original variables as possible. Because each principal component contains information from all the variables, it allows important patterns in the data to be summarized in a small number of dimensions, in this case a two-dimensional graph. The graph of the grenade data (Figure 8) shows how well the first two principal components separate the grenades by color. In the graph, two of the yellow grenades are clustered with the green grenades. This indicates that more information than the first two principal components contain is required to completely distinguish between the grenades. While the higher dimensionality ${ }^{2}$ of the problem precludes illustrating the separation graphically, the discriminant analysis showed that complete separation (discrimination) can be achieved (i.e. $100 \%$ correct classification).

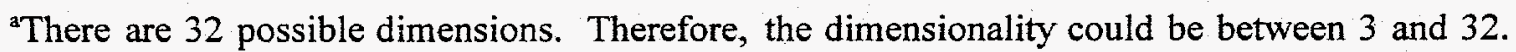




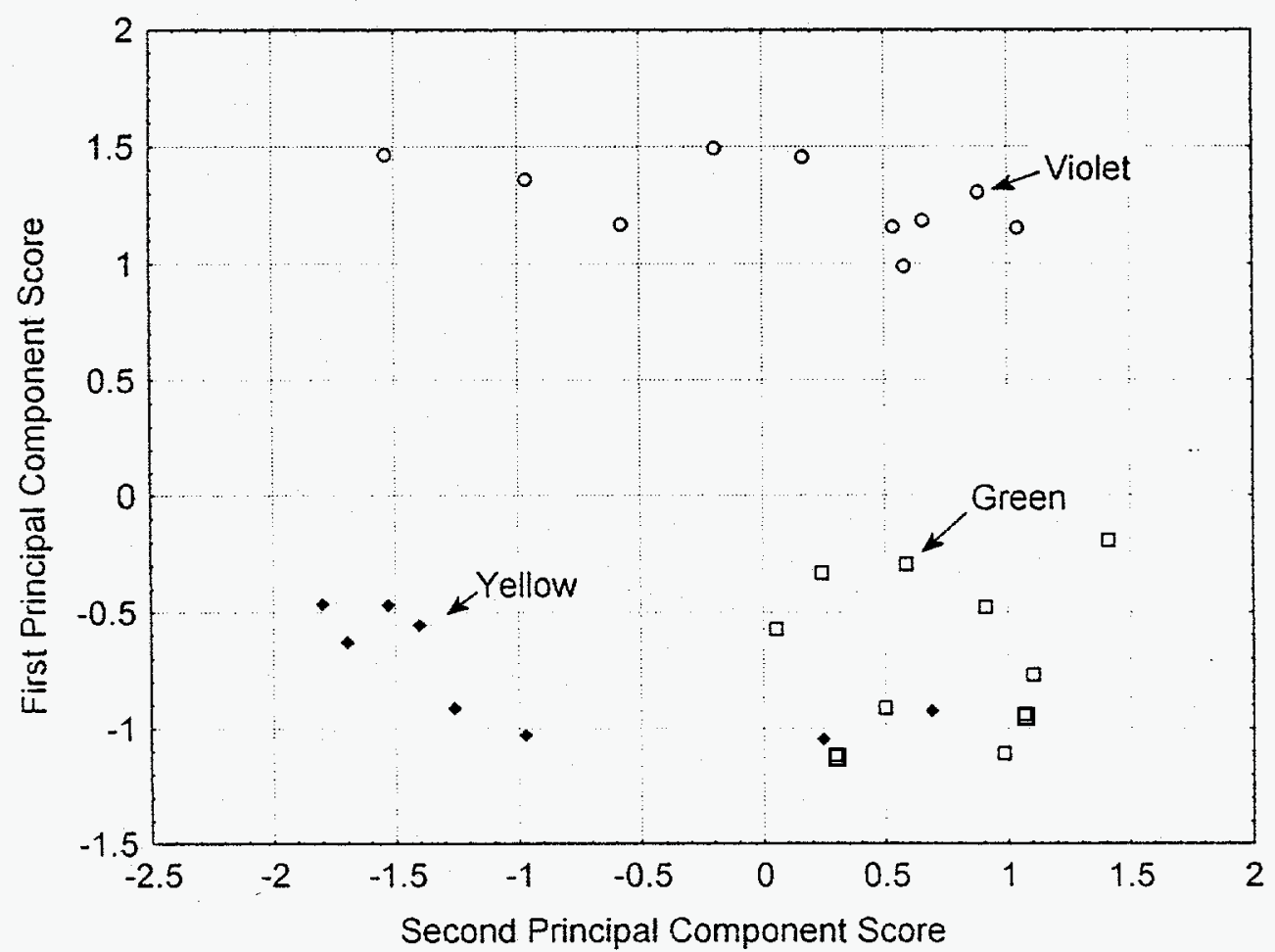

Figure 8. First two principal components for grenade data. 


\section{SUMMARY}

A noncontacting, long-standoff inspection system for container fill identification is being developed at the Idaho National Engineering Laboratory. During FY-93 a number of improvements were made to the system to simplify its operation, enhance its performance, and make it more compact. Software improvements included development of a graphical user interface, which makes execution of the computer program that controls data acquisition simpler and more understandable, and establishment of a software configuration management plan. A number of hardware modifications were also made to the inspection system. Most parameters defining the data acquisition sequence are now under computer control, resulting in fewer operational errors, better documentation, and speedier measurements. A non-fiber optical head for the laser vibrometer was acquired, making the vibrometer easier to operate and improving its performance.

A finite element numerical model was developed to study the natural frequency response of a 155 $\mathrm{mm}$ projectile. A variety of parametric calculations were used to study the effects of geometry and fill material. Finite element analysis demonstrated that there is a strong correlation between predicted and measured frequency components that could be used to predict acoustic signatures for chemical filled shells. The numerical results indicate that the lowest axial vibration mode $(m=1)$ for the 2-lobe ringing mode $(n=2)$ is most susceptible to the effects of filling. For this mode, including a paraffin filler increased the natural frequency by approximately $12 \%$, whereas a $10 \%$ increase in the projectile wall thickness increased the frequency by approximately $5 \%$. For this same mode, the effects of the internal burst tube were insignificant. The numerical results are useful in determining appropriate locations on the projectile surface for data collection. Obviously, optimal locations differ for different vibrational modes, thus it may be necessary to select a less than optimal location (or possibly multiple locations) to sense multiple modes. To the extent comparisons are possible, excellent agreement was observed between numerical and experimental results. Continued efforts will address integration of finite element analysis in developing a robust classification algorithm.

The KNN classification tree was proven to be a useful method for classifying objects measured with the laser acoustic system, as indicated by cross-validation results. These results are somewhat optimistic in that the classification of actual new objects (rather than reclassification of those in the training set) will likely result in somewhat lower correct classification rates. Still, the method shows great promise. Future efforts will be directed at actual classification of new objects, and further investigation of optimal data preprocessing and data reduction procedures.

Tests at Dugway demonstrated that not only can the NAUSA system easily collect data from muddy munitions, but mud improves the signal-to-noise ratio. As expected, the added loading from the mud affected the vibration signatures. The ability of the NAUSA system to collect data from muddy munitions can be attributed to its ability to make measurements without "touching" the target. With mud on the surfaces, a contacting system could not have obtained the measurements needed to classify these munitions. Data collected from the grenades indicated that the NAUSA system can differentiate between various smoke-filled grenades. Due to the small size of the smoke grenades measured, transducer surface loading and coupling efficiency would pose problems for a contacting system that are not encountered by the noncontacting system.

The NAUSA system offers many technical advantages over current systems. It is noncontacting and has a long standoff capability, which contributes substantially to inspection safety during the interrogation 
of hazardous objects such as munitions. It can obtain measurements from a wide variety of surfaces and surface conditions. Thin-wall structures and non-ferromagnetic materials pose no problems in obtaining acoustic signatures for munition classification. The most conspicuous drawback of the current system is its size. A goal for future development efforts is to develop a smaller, more portable inspection system. 


\section{REFERENCES}

1. Computer code PATRAN, PDA Engineering, Costa Mesa, CA, 1992.

2. Computer code ABAQUS, Hibbitt, Karlsson, and Sorenson, Inc., Providence, RI, 1992.

3. A. Kalnins and C. L. Dym, Vibration - Beams, Plates, and Shells, Dowden, Hutchinson \& Ross, Inc., Stroudsburg, Pennsylvania, 1976, p. 184.

4. P. D. Heimdahl, "Theoretical Analysis of Acoustic Spectroscopy as a Method of Determining Contents of Artillery Projectiles," unpublished study, Los Alamos National Laboratory.

5. SAS/STAT User's Guide, Release 6.03 Edition, SAS Institute Inc., Cary, North Carolina, 1988. 